\title{
Synaptic Basis for Contrast-Dependent Shifts in Functional Identity in Mouse V1
}

\author{
(D) Molis Yunzab,, ${ }^{1,2^{*}}$ (D) Veronica Choi, ${ }^{3 *}$ - Damish Meffin, ${ }^{1,2}$ CShaun L. Cloherty, ${ }^{1,2,4}$ Nicholas J. Priebe, ${ }^{3^{\dagger}}$ \\ and Michael R. Ibbotson ${ }^{1,2^{\dagger}}$
}

https://doi.org/10.1523/ENEURO.0480-18.2019

${ }^{1}$ National Vision Research Institute, Australian College of Optometry, Carlton, Victoria 3053, Australia, ${ }^{2}$ Department of Optometry and Vision Sciences, University of Melbourne, Parkville, Victoria 3010, Australia, ${ }^{3}$ University of Texas Austin, Centre for Learning and Memory, Austin, TX 78712, and ${ }^{4}$ Department of Physiology, Monash University, Clayton, Victoria 3800, Australia

\begin{abstract}
A central transformation that occurs within mammalian visual cortex is the change from linear, polarity-sensitive responses to nonlinear, polarity-insensitive responses. These neurons are classically labelled as either simple or complex, respectively, on the basis of their response linearity (Skottun et al., 1991). While the difference between cell classes is clear when the stimulus strength is high, reducing stimulus strength diminishes the differences between the cell types and causes some complex cells to respond as simple cells (Crowder et al., 2007; van Kleef et al., 2010; Hietanen et al., 2013). To understand the synaptic basis for this shift in behavior, we used in vivo whole-cell recordings while systematically shifting stimulus contrast. We find systematic shifts in the degree of complex cell responses in mouse primary visual cortex (V1) at the subthreshold level, demonstrating that synaptic inputs change in concert with the shifts in response linearity and that the change in response linearity is not simply due to the threshold nonlinearity. These shifts are consistent with a visual cortex model in which the recurrent amplification acts as a critical component in the generation of complex cell responses (Chance et al., 1999).
\end{abstract}

Key words: complex cell; in vivo whole-cell recording; phase sensitivity; primary visual cortex; visual system

\section{Significance Statement}

The discovery of simple and complex cells in the primary visual cortex (V1) has been fundamental to our understanding of visual processing. While both cell types are orientation selective, simple cells are spatial phase sensitive while complex cells are phase invariant. Extracellular recordings have shown that the responses of complex cells become phase sensitive at lower stimulus contrasts, suggesting more flexibility in processing mechanisms than previously thought. The mechanism by which this flexibility arises is not understood. Using in vivo whole-cell recordings, we demonstrated that the flexibility in phase sensitivity is also apparent in the subthreshold responses of mouse V1 cells, suggesting that the effect arises from active cortical recurrent network activity and not from passive spiking threshold mechanisms.

\section{Introduction}

The receptive fields (RFs) of cells in the primary visual cortex (V1) are classified as either simple or complex

\footnotetext{
Received December 11, 2018; accepted February 27, 2019; First published March 20, 2019.

The authors declare no competing financial interests.

Author contributions: M.Y., N.J.P., and M.R.I. designed research; M.Y. and V.C. performed research; M.Y., V.C., H.M., S.L.C., N.J.P., and M.R.I. analyzed data; M.Y., V.C., N.J.P., and M.R.I. wrote the paper.
}

based on their spatial organization (Hubel and Wiesel, 1962; Henry, 1977). Simple cell RFs have segregated subfields that respond to either brightness increments $(\mathrm{ON})$ or decre-
This work was supported by the Australian Research Council Centre of Excellence for Integrative Brain Function Grant CE140100007, the National Health and Medical Research Council Grant GNT0525459, the National Health Institutes Grant EY 020592 and the L.E.W. Carty Charitable Fund \& Lions Foundation of Victoria.

${ }^{*}$ M.Y. and V.C. contributed equally to this work. 
ments (OFF); complex cells do not have clearly segregated ON and OFF subfields (Hubel and Wiesel, 1962; Gilbert, 1977; Henry, 1977; Hammond and Ahmed, 1985; Spitzer and Hochstein, 1988; Mechler and Ringach, 2002; Priebe et al., 2004; Hietanen et al., 2013). The Hubel and Wiesel hierarchical model proposed that convergent synaptic inputs are responsible for these transformations in two stages (Hubel and Wiesel, 1962): thalamic relay cells, displaced along an oriented axis, converge on simple cells, generating orientation selectivity, then simple cells converge on complex cells to provide polarity invariance.

The distinction between simple and complex cells is related to neuronal laminar position and synaptic connectivity in some mammals (Ringach et al., 2002; Martinez et al., 2005; Williams and Shapley, 2007). Simple cells are found more often in cortical layers that receive thalamocortical connections, while complex cells are found in layers with dense recurrent cortical connectivity. The differences between simple and complex cell RFs may reflect a general process in which cortical circuits generalize selectivity by amplifying inputs. While cortical amplification has previously been hypothesized to increase selectivity (Ben-Yishai et al., 1995; Douglas et al., 1995; Somers et al., 1995), it is also possible for it to generalize selectivity by integrating inputs with distinct RFs. We examined whether simple and complex cell responses in V1 exhibited signatures of this amplification.

One quantitative method to distinguish simple and complex cells depends on the relative modulation of responses to drifting sinusoidal gratings (Skottun et al., 1991). When stimulated with high-contrast drifting gratings, simple cell responses modulate as the grating moves across the distinct ON and OFF subfields. In contrast, complex cells respond to all phases of the drifting gratings. Studies have demonstrated that the ratio $\left(F_{1} / F_{0}\right)$ of the modulated spiking component $\left(F_{1}\right)$ to the unmodulated component $\left(F_{0}\right)$ forms a bimodal distribution, suggesting two classes of V1 neurons (Maffei and Fiorenti, 1973; Movshon et al., 1978; De Valois et al., 1982; Skottun et al., 1991). While this difference between cell classes is clear when the stimulus strength is high, reducing stimulus strength diminishes the differences between the cell types. In particular, low contrast gratings evoke modulated responses in many complex cells (cat: Crowder et al., 2007; van Kleef et al., 2010; monkey: Henry and Hawken, 2013; Cloherty and Ibbotson, 2015; Meffin et al., 2015).

The mechanism underlying this change in spiking modulation ratio is not understood but there are two candidate models. The first model suggests that modulations in response to low contrast stimuli emerge due to the "iceberg" effect in which not all synaptic responses are converted into spikes (Carandini and Ferster, 2000; Mechler

${ }^{\dagger}$ N.J.P. and M.R.I. contributed equally to this work.

Correspondence should be addressed to Michael R. Ibbotson at mibbotson@nvri.org.au

https://doi.org/10.1523/ENEURO.0480-18.2019

Copyright (C) 2019 Yunzab et al.

This is an open-access article distributed under the terms of the Creative Commons Attribution 4.0 International license, which permits unrestricted use, distribution and reproduction in any medium provided that the original work is properly attributed. and Ringach, 2002; Priebe et al., 2004). In this model the subthreshold synaptic modulation ratio $\left(V_{1} / V_{0}\right)$ should not depend on contrast. Alternatively, there may be a shift in the synaptic inputs to $V 1$ neurons in which the $V_{1} V_{0}$ ratio increases as the contrast decreases. A cortical model in which the amplification acts to integrate inputs with distinct spatial preferences predicts this specific change in synaptic input.

To distinguish these possibilities, we performed in vivo whole-cell recordings in mouse $V 1$. Both the $V_{1} N_{0}$ and the $F_{1} / F_{0}$ ratios increased as contrast was reduced indicating that a change in synaptic drive is the more likely explanation for the altered modulation responses of complex cells. We have demonstrated that the circuitry leading to spatial phase invariant responses in visual cortex depends on the strength of visual drive. This observation is consistent with a scheme of complex cell generation in which the recurrent inputs in the visual cortex act as amplifiers, generating linear or nonlinear responses when input gain is low or high, respectively (Chance et al., 1999).

\section{Materials and Methods}

\section{Electrophysiology}

Recordings were made from anaesthetized C57BL/6 mice of both sexes aged five to twelve weeks. All experiments were performed according to the National Health and Medical Research Council's Australian Code of Practice for the Care and Use of Animals for Scientific Purposes. All experimental procedures were approved by Animal Ethics Committees of the University of Melbourne, or by the Institutional Animal Care and Use Committee of the University of Texas at Austin. Mice were anesthetized with intraperitoneal injections of chloroprothixene $(10 \mathrm{mg} /$ $\mathrm{kg})$ followed by urethane $(1 \mathrm{~g} / \mathrm{kg})$. Animals also received an injection of dexamethasone $(2 \mathrm{mg} / \mathrm{kg})$ to reduce brain edema. The level of anesthesia was monitored using the electrocardiogram (ECG) and repeated toe-pinches throughout the experiment. Body temperature was monitored and maintained at $37^{\circ} \mathrm{C}$ using an auto-regulating heating blanket. A tracheotomy was performed to ensure a clear airway. A craniotomy $\sim 1 \times 2.5 \mathrm{~mm}$ was performed over V1 in one hemisphere and the dura mater retracted.

Intracellular responses were obtained in mice via blind recordings with a whole-cell configuration in vivo as previously described (Ferster and Jagadeesh, 1992; Margrie et al., 2002; Priebe et al., 2004; Tan et al., 2011). Patch pipettes with tip resistances of 8-10 MOhm were pulled from borosilicate glass capillaries $(1.2 \mathrm{~mm}$ outer diameter, 0.7 mm inner diameter; KG-33, King Precision Glass). A silver chloride coated silver wire was inserted into the pipette, which was filled with $135 \mathrm{mM}$ K-gluconate, $4 \mathrm{mM}$ $\mathrm{NaCl}, 0.5 \mathrm{mM}$ EGTA, $2 \mathrm{mM}$ MgATP, $10 \mathrm{mM}$ phosphocreatine disodium, and $10 \mathrm{mM}$ HEPES; $\mathrm{pH}$ adjusted to 7.3 with $\mathrm{KOH}$ (Sigma-Aldrich). A silver-silver chloride wire was inserted as a reference electrode into muscles near the base of the skull. The craniotomy as well as the reference electrode was covered with $4 \%$ agarose in normal saline to keep the cortex moist and to reduce changes in the surrounding fluid and concomitant changes in associated junction potentials. An Axoclamp 2B patch-clamp amplifier was 
used in current clamp to record from neurons 150-600 $\mu \mathrm{m}$ below the surface of the cortex. The voltage was digitized and recorded with custom software (Labview, National Instruments), which also sent instructions to a separate stimulus-generation computer.

\section{Visual stimuli}

Visual stimuli were generated using the Psychophysics toolbox for MATLAB (The MathWorks Inc.) and were presented on a calibrated CRT monitor (Sony GDM-F520, $100 \mathrm{~Hz}$ non-interlaced refresh rate, $1280 \times 1024$ pixels, $25 \mathrm{~cd} / \mathrm{m}^{2}$ mean luminance). The viewing distance for all recordings was $30 \mathrm{~cm}$. For each recorded cell we measured its orientation, spatial frequency (SF), and temporal frequency (TF) preferences, as well as its RF location and size using drifting sinusoidal gratings. For example, to determine orientation preference, sinusoidal gratings were presented at eight different orientations $\left(0^{\circ}, 22.5^{\circ}\right.$, $\left.45^{\circ}, 67.5^{\circ}, 90^{\circ}, 112.5^{\circ}, 135^{\circ}, 157.5^{\circ}\right)$. After $0.5 \mathrm{~s}$ of presentation of each orientation, gratings moving in the opposite direction were presented and followed with $0.5 \mathrm{~s}$ of gray screen (at the mean luminance of the prior grating). The optimal tuning parameters were determined online and then applied to the experimental stimuli. The contrast of the grating was defined as: Michelson contrast = $\left.\left[\left(\operatorname{Lum}_{\max }-\mathrm{Lum}_{\text {min }}\right) / \mathrm{Lum}_{\max }+\mathrm{Lum}_{\text {min }}\right)\right] \times 100$, where $\mathrm{Lum}_{\max }$ and $\mathrm{Lum}_{\text {min }}$ are the maximum and minimum luminance of the grating.

Two types of experimental stimuli were used: drifting sinusoidal gratings and sinusoidally modulated contrastreversing gratings. Stimuli were presented at the optimal $\mathrm{TF}, \mathrm{SF}$, and orientation of the recorded cell in a circular aperture the size of its excitatory RF. Drifting gratings with contrast levels ranging between $4 \%$ and $100 \%$ were presented in pseudorandom order interleaved with 1-s presentations of a blank (mean luminance) screen. Each grating was presented for $3 \mathrm{~s}$ with the first and last $0.5 \mathrm{~s}$ stationary, and drifting for the $2 \mathrm{~s}$ in between. Trials were repeated as many times as the stability of the recording would allow. Contrast-reversing gratings were presented at 8 different spatial phases $\left(0^{\circ}, 45^{\circ}, 90^{\circ}, 135^{\circ}, 180^{\circ}, 225^{\circ}\right.$, $270^{\circ}, 315^{\circ}$ ). Depending on the recording stability, various combinations of contrast between $8 \%$ and $100 \%$ were tested. Each stimulus presentation consisted of a grating presented for $0.5 \mathrm{~s}$ with a steady contrast, $2 \mathrm{~s}$ presented with sinusoidally modulated contrast, and another $0.5 \mathrm{~s}$ with steady contrast.

\section{Data analysis}

The resting membrane potential $\left(\mathrm{V}_{\text {rest }}\right)$ of a patched cell, measured as the responses to a blank screen $(0 \%$ contrast), ranged from -40 to $-80 \mathrm{mV}$. To examine the subthreshold membrane potential modulation, spikes were removed from the raw records before analysis using a 5 ms median filter (Jagadeesh et al., 1997). The modulation ratios for membrane potential $\left(V_{1} N_{0}\right)$ and spiking rate $\left(F_{1} / F_{0}\right)$ to drifting gratings were calculated as previously described in Priebe et al. (2004). For contrast-reversing gratings, the modulation ratios for membrane potential and spiking rate were calculated as $V_{2} / V_{1}$ and $F_{2} / F_{1}$, respectively. Cycle-averaged responses were measured by aligning each response cycle, excluding the first cycle. The mean and standard error of the membrane potential and spiking rate were calculated at each time point of the cycle-averaged response. As in Priebe et al. (2004), the mean membrane potential $\left(V_{0}\right)$ and spiking rate $\left(F_{0}\right)$ are based on the differences between the responses during a stimulus and the spontaneous responses during a blank screen of the same time. Fourier coefficients at the fundamental frequency of the stimulus grating $\left(V_{1}\right.$ for membrane potential, $F_{1}$ for spiking rate) and at twice the stimulus input $\left(V_{2}\right.$ for membrane potential, $F_{2}$ for spiking rate) for each cycle-averaged response were extracted using the FFT function in MATLAB (The MathWorks Inc.). $A$ perfect half-wave rectified spiking rate response is expected to have an $F_{1} / F_{0}$ ratio of 1.57 . We found two cells with $F_{1} / F_{0}$ ratios above 1.57 at high stimulus contrasts, but this was due to low $F_{0}$ values created by subtracting the high spontaneous spiking rate from the evoked spiking rate. All cells showed significant increases in mean spiking rate $\left(F_{0}\right)$ relative to the spontaneous spiking rate $(p$ $<0.05$, one-sided $t$ tests). One cell with a modulation ratio $\left(\mathrm{F}_{1} / \mathrm{F}_{0}\right)$ of 2.67 had a relatively high spontaneous spiking rate $(6.9$ spikes/s) compared to its evoked spiking rate (9.6 spikes/s). The other cell with $F_{1} / F_{0}$ of 1.98 showed a relatively high spontaneous spiking rate (1.2 spikes/s) compared to its evoked spiking rate ( $6.5 \mathrm{spikes} / \mathrm{s})$. Both cells showed significant increases in evoked $\mathrm{F}_{1}$ amplitude with a clear response to the drifting grating stimulus $(p=$ 0.0002 and $p<0.0001$, one-sided $t$ tests). Error bars were generated by projecting the cycle-by-cycle estimate of modulation amplitude and phase onto the mean phase and amplitude vector in complex space.

\section{Model}

Each neuron in the network model receives feedforward and recurrent input and is based on the rate model developed by Chance et al. (1999). The activity of neuron $i$, is modeled using a simple rate model equation that includes a threshold nonlinearity:

$$
\begin{gathered}
\tau_{v} \frac{d v_{i}}{d t}=l_{i}^{\text {ff }}+l_{i}^{\text {rec }}-v_{i} \\
r_{i}=\left\lfloor v_{i}-v_{\text {thresh }}\right\rfloor+
\end{gathered}
$$

where $I_{i}^{f f}$ and $I_{i}^{\text {rec }}$ represent the feedforward and recurrent inputs. We use a time constant, $\tau_{r}$ of $20 \mathrm{~ms}$ and a positive voltage threshold $\left(v_{\text {thresh }}\right)$. The feedforward input is equal to a half-wave rectified sinusoidal modulation, where each network neuron has a random preferred spatial phase $\left(\phi_{i}\right)$.

$$
l_{i}^{f f}=\rho \sin \left(\frac{2^{*} \pi t}{500}+\phi_{i}\right)
$$

The recurrent input to model neuron $i$ is given by:

$$
l_{i}^{\text {rec }}=\frac{\left(1-\rho_{i}\right)}{N} \sum r_{i}
$$

The degree of recurrent and feedforward input is set by the value of $\rho_{i}$, which was randomly set between 0 and 1 , 
A

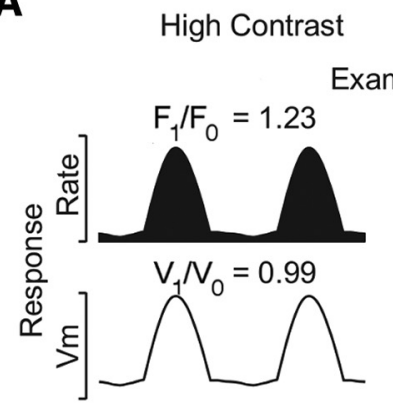

Example cell 1

Low Contrast

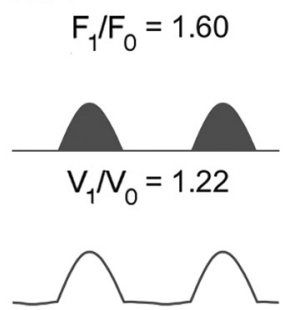

Example cell 2
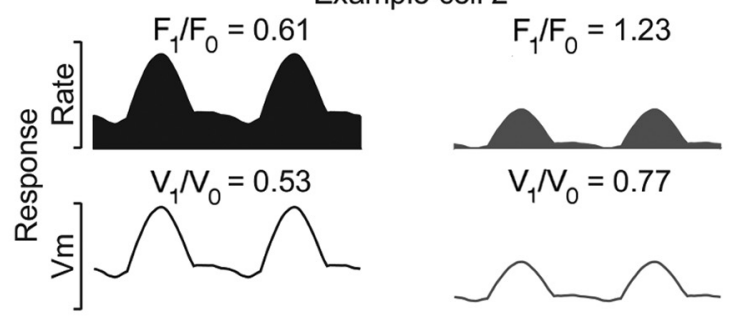

Example cell 3

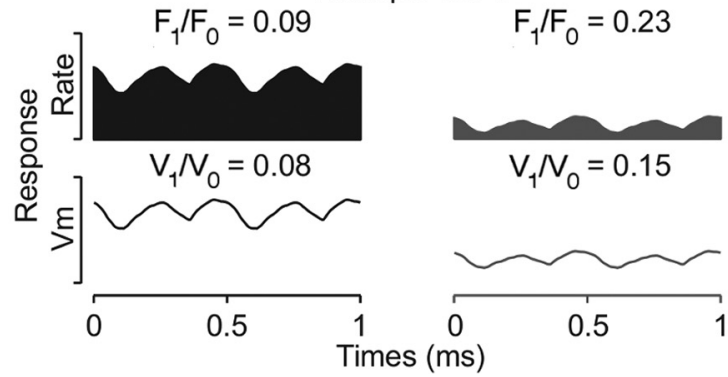

B

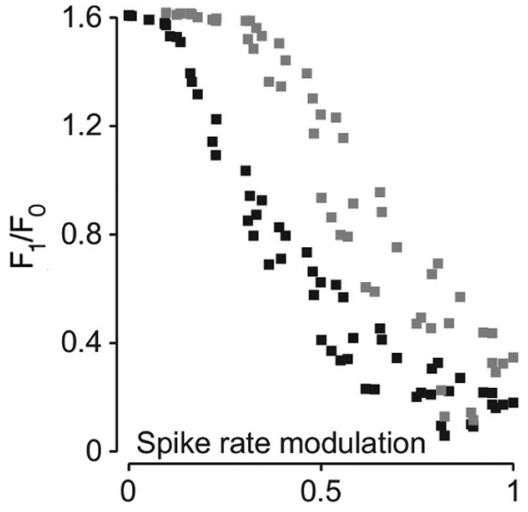

C Proportion recurrent input $(\rho)$

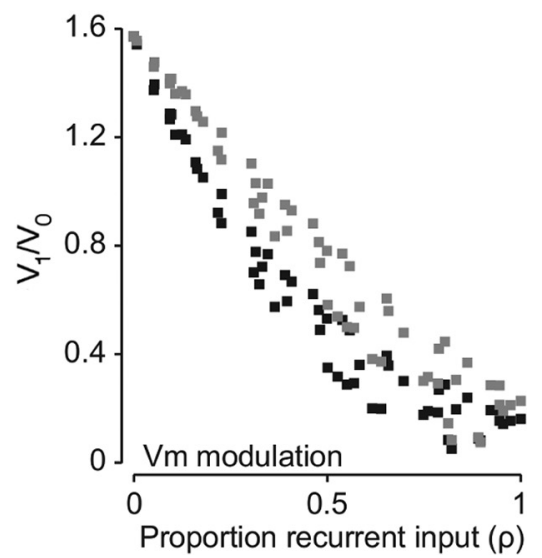

Figure 1. Model for contrast-dependent transformation between simple and complex cells in mammalian $\mathrm{V} 1$ neurons. $\boldsymbol{A}$, Example of a model cell with a modulation ratio $\left(F_{1} / F_{0}\right)$ that is greater than 1 (example cell 1, top), a model cell with $F_{1} / F_{0}$ between 0.5 and 1 (example cell 2 , middle), and a cell with $F_{1} / F_{0}$ below 1 (example cell 3 , bottom) at high contrasts. The top row represents the spiking rate of the cell and the bottom row is the underlying membrane potential of the cell. The black trace to the left shows responses of the cell to high contrasts and the light grey trace to the right shows responses to low contrasts. $\boldsymbol{B}$, Black dots represent spiking rate at high contrast and grey dots represent spiking rate at low contrast. As the proportion of recurrent input increases (high contrast) the modulation ratio derived from the spiking rate $\left(F_{1} / F_{0}\right)$ decreases. $\boldsymbol{C}$, same as $\boldsymbol{B}$ but for membrane potential.

with zero reflecting all recurrent input and one reflecting all feedforward input.

\section{Results}

We first explored a model of the transformation between simple and complex cells to guide our experiments based on the architecture from Chance et al. (1999). They used a rate model to demonstrate that the degree of simple cell and complex cell behavior is related to the amount of recurrent circuitry in the network. We implemented their model with two changes. First, each neuron received a random degree of feedforward input $\left(\rho_{i}\right)$ so that we would observe both simple and complex cells within the same network. Second, we included a non-zero threshold to model the threshold nonlinearity between the input and output. Both simple and complex cells emerge from this network model, as seen in the modulation ratio to a drifting grating (Fig. 1A). Simple cells respond at one phase of the stimulus and have a modulation ratio that is $>1$ (Fig. 1A, top row). Network complex cells exhibit a response that varies little with the drifting grating and are characterized by a modulation ratio $<1$ (Fig. $1 A$, bottom row). Importantly in this network simulation we can view neurons that exhibit combinations of linear and nonlinear components (Fig 1A, middle row) and therefore have a modulation ratio between 0.5 and 1 .

As the emergence of complex cells in this model depends on the degree of recurrent amplification, we hypothesized that reducing the input strength would impact the modulation ratios of network neurons, and thus the degree of generalization across spatial phase. Indeed, we find that reducing input strength, or visual contrast, leads to systematic increases in the modulation ratios of network neurons (Fig. 1B). The modulation ratios of model neurons shift to higher values as the input strength is reduced, even switching neurons that would be classified 

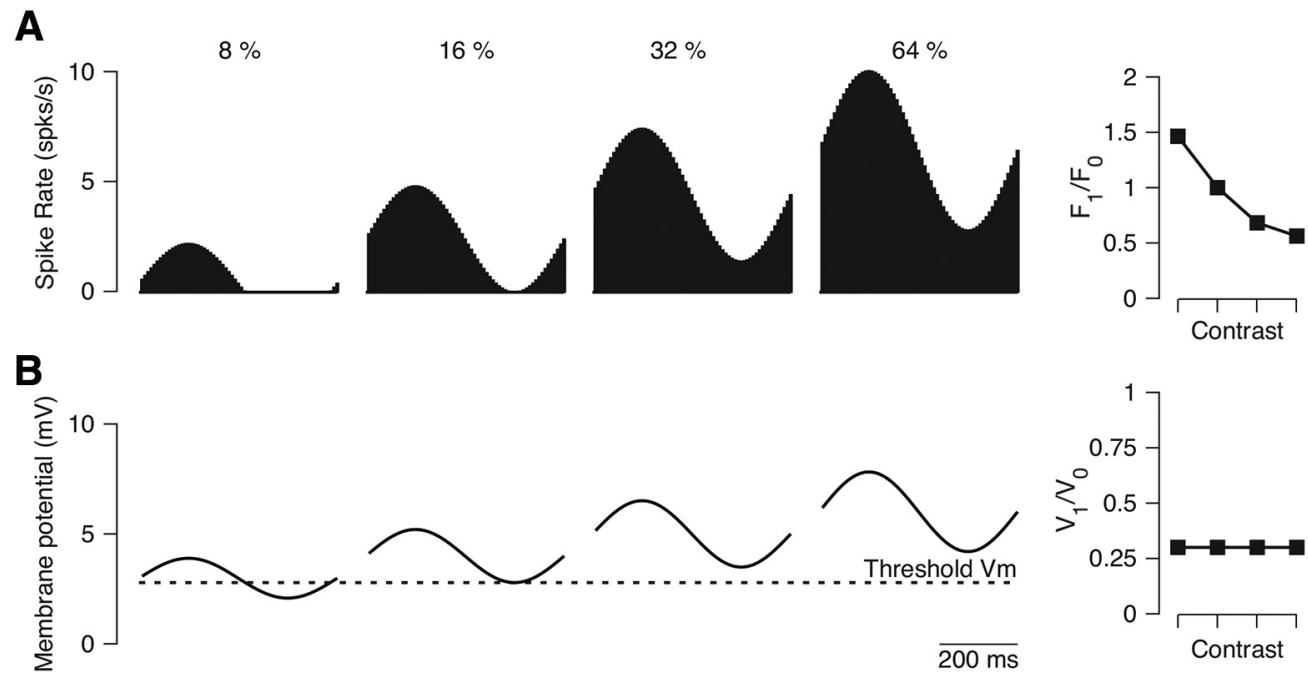

Figure 2. Threshold nonlinearity model ("iceberg" model) for a contrast-dependent transformation between simple and complex cells in mammalian V1 neurons. $\boldsymbol{A}$, Spiking responses of a model neuron relative to contrast. As contrast increases, the spiking rate of the model neuron increases (right). The modulation ratio $\left(F_{1} / F_{0}\right)$ decreases with increasing contrast due to relatively large increases in the mean spiking rate $\left(F_{0}\right)$ compared to the $\mathrm{F} 1$ component. $\boldsymbol{B}$, Membrane potential responses of the model neuron relative to contrast. As the contrast increases, the amplitude of the membrane potential increases. The matched increases in the $V_{0}$ and $V_{1}$ components of the responses generate a steady modulation ratio $\left(V_{1} / V_{0}\right)$ at all contrasts.

as complex for high contrast to being simple at low contrast (Fig. 1A, middle row). Contrast-dependent changes in the modulation ratio could therefore reflect the amplification structure of the model visual cortex. There are two components that contribute to the increase in modulation ratio with contrast. First, the observed increase in the network modulation ratio with contrast depends on the voltage threshold $\left(V_{\text {thresh }}\right)$. If the voltage threshold is set to 0 then no change in modulation ratio occurs with changes in contrast (data not shown). Second, there are systematic increases in the synaptic modulation ratio (Fig. 1C) as contrast is reduced. This simple model demonstrates how the cortical circuitry, acting as an amplifier, could generate spatially invariant responses and demonstrates that the degree of the spatial invariance depends on the input strength.

There are other possible models that could explain the shift in modulation ratio due to changes in contrast. One alternative possibility is an iceberg effect where not all synaptic responses are converted into spiking activities (Carandini and Ferster, 2000; Mechler and Ringach, 2002; Priebe et al., 2004). For a high contrast stimulus, the synaptic input is sufficient to evoke spiking responses at all phases (Hietanen et al., 2013), whereas for a low contrast stimulus, the synaptic input falls below threshold and is only sufficient to evoke spiking responses for a subset of phases (Fig. 2A). In this model, the modulation ratio of the synaptic input $\left(V_{1} / N_{0}\right.$ : the modulation ratio of the membrane potential) does not vary (Fig. 2B); instead the change in the spiking modulation ratio is due to the threshold nonlinearity. This explanation for the observed changes in the spike modulation ratio with contrast proposes that the underlying membrane potential modulation ratio is fixed and the changes observed at the level of spiking emerge from the threshold nonlinearity.

To examine whether signatures of these models exist in V1 neuron responses we measured the degree to which the modulation ratio of the synaptic input varies with contrast. Previous experimental reports have demonstrated that the spiking modulation ratio of $\mathrm{V} 1$ neurons is contrast dependent, which matches the pattern shown in the model, i.e., responses become more simple-like as contrast declines. However, these records do not differentiate between synaptic changes from the network and changes that may exclusively emerge from threshold nonlinearity. To determine whether the change in the spiking modulation ratio is due to threshold or synaptic mechanisms, we recorded intracellularly from V1, giving us access to both the underlying membrane potential as well as the spiking rate in response to gratings. We recorded from 20 cells with drifting gratings and 21 cells with contrast reversing gratings in 20 urethane-anaesthetized mice.

\section{Responses to drifting gratings}

Based on responses to drifting sinusoidal gratings, mouse V1 neurons show the same separation into simple and complex cells as cats and primates (Niell and Stryker, 2008). We classified cells as simple by the large modulation of spiking rate $\left(F_{1} / F_{0}>1\right)$ to a drifting grating stimulus. The underlying membrane potential of these neurons also exhibited large modulations when stimulated at the preferred orientation, SF and TF (100\% contrast; Fig. 3). Membrane potential fluctuations were separated from spiking rate by identifying the spike times and removing them from the membrane potential traces using a median filter (see Materials and Methods). Both the raw response and the trial-averaged membrane potential for the simple cell in Figure 3 are highly modulated at the input fre- 

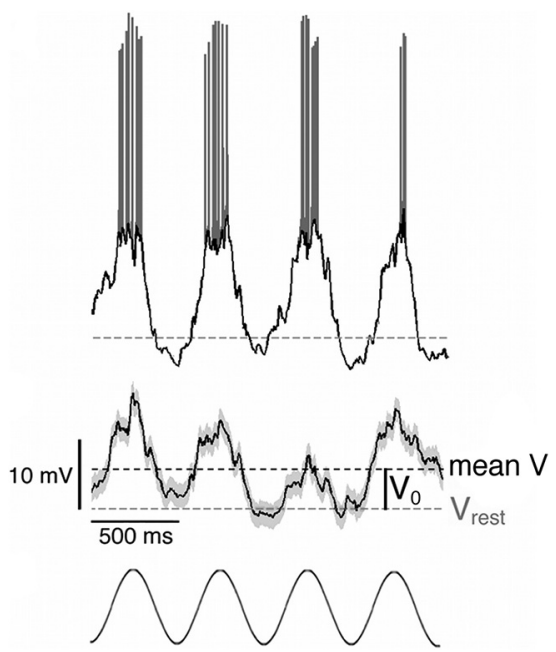

Figure 3. Intracellular responses of a mouse V1 simple cell to a drifting sinusoidal grating. Top panel: raw response to a sinusoidal grating with $100 \%$ contrast moving at $2 \mathrm{~Hz}$ at the cell's preferred orientation. The black trace represents the subthreshold membrane potential; spikes are shown in grey. The broken line indicates the resting membrane potential of the cell: -48.5 $\mathrm{mV}$. Middle panel: trial-averaged (5 repeats) subthreshold voltage trace for the same cell, including the data in the top panel. Spikes are removed from voltage traces prior to averaging by calculating the derivative of the membrane potential and excluding rapid voltage excursions based on a derivative threshold. The grey shading shows the standard error of the mean. The mean membrane potential (mean $\mathrm{V}$ ) and resting membrane potential $\left(V_{\text {rest }}\right)$ for the trial-averaged subthreshold responses are shown respectively as dark grey and light grey broken lines. The voltage difference between the mean and resting membrane potential is defined as $\mathrm{V}_{0}$. Bottom panel: $\mathrm{A}$ visual representation of 4 cycles of the sinusoidal grating stimulus moving at $2 \mathrm{~Hz}$ (2 s in total).

quency and phase-locked to the sinusoidal grating stimulus (Fig. 3, bottom).

Previous work has demonstrated that the classification of simple cells does not vary with contrast in the cat and primate (cat: Crowder et al., 2007; van Kleef et al., 2010; monkey: Henry and Hawken, 2013; Cloherty and lbbotson, 2015; Meffin et al., 2015). We first examined whether this is also true in mouse $\mathrm{V} 1$ by measuring the changes in the modulation ratios for spiking rate $\left(F_{1} / F_{0}\right)$ and membrane potential $\left(V_{1} / V_{0}\right)$ of individual simple cells with contrast (Fig. 4A). For simple cells, $V_{1} N_{0}$ did vary with contrast but the $F_{1} / F_{0}$ was consistently higher than unity, indicating that simple cell classification does not depend on contrast (Fig. 4A). Across our sample population we found that the subthreshold modulation ratio $\left(V_{1} / N_{0}\right)$ of simple cells often increased with decreasing contrast but this change was not statistically significant $(n=13, p>$ 0.05 , one-sided $t$ test; Fig. $5 A$, red symbols). This result is consistent with results from an earlier study, in which simple cells in cat $V_{1}$ showed increased $V_{0}$ and $V_{1}$ as contrasts decreased (Carandini and Ferster, 1997). Despite those subthreshold changes, however, the $F_{1} / F_{0}$ ratio was consistently above unity for simple cells at low and high contrasts (Fig. 5B). Therefore, the simple cell population remains highly phase sensitive at both the membrane potential and spiking output levels for all contrasts.

We next examined how contrast alters the modulation ratio of complex cells in mouse V1. As found in other mammals, complex cells modulate more at low contrasts than high contrasts (cat: Crowder et al., 2007; van Kleef et al., 2010; monkey: Henry and Hawken, 2013; Cloherty and Ibbotson, 2015; Meffin et al., 2015). We found a range of contrast-dependent shifts in the modulation ratios, which demonstrate that synaptic mechanisms are involved in this process. For some complex cells modulations in response amplitude are clearly evoked across all contrasts at the level of both the membrane potential and spiking rate (Fig. 4B). Measures of the modulation ratios of the membrane potentials systematically increase as contrast decreases. This shift is matched by a commensurate increase in the $F_{1} / F_{0}$ ratios. For both spiking rate and membrane potential responses, the mean responses $\left(F_{0}\right.$ and $\left.V_{0}\right)$ dominate the modulation amplitudes $\left(F_{1}\right.$ and $\left.V_{1}\right)$ at high contrasts (Fig. 4B). As the contrast decreases, however, the differences between these two parameters declines and results in increased modulation ratios. This trend is especially prominent in the membrane potential responses in which $V_{1}$ remains largely unchanged compared to $\mathrm{V}_{0}$. When considering the complex cell and the simple cell examples together, it is noticeable that similar membrane potential characteristics in the two example cells (Fig. $4 A, B)$ are observed at higher contrasts $(32 \%$ and $64 \%)$. Both cells show substantial modulations of the fundamental frequencies of the input $\left(V_{1}\right)$ that are well above the resting membrane potential, which result in the $\mathrm{V}_{0}$ component being larger than the $\mathrm{V}_{1}$ component. However, the spiking responses show different response characteristics in the two cells: cell $A$ has a larger $F_{1}$ component at high contrasts, whereas cell $B$ has a larger $\mathrm{F}_{0}$ component (Fig. $4 A, B$ ). As a result, cell $A$ has an $F_{1} / F_{0}$ $>1$ and is therefore classified as a simple cell, whereas cell $B$ is classified as a complex cell because it has an $F_{1} / F_{0}<1$. These observations suggest that the dichotomy between simple and complex cells based on spiking modulation ratios with high stimulus strengths does not directly translate to corresponding distinctions in the membrane potential responses. The differences in the $F_{1} / F_{0}$ ratios in the two cells are likely the result of nonlinear threshold transformations from the membrane potentials to the spiking outputs (Priebe et al., 2004).

At the population level, changes in the $F_{1} / F_{0}$ (spiking rate) and $V_{1} N_{0}$ (membrane potential) ratios of complex cells at high and low contrasts have characteristics similar to the responses of the synaptic model. The scatter plots of both $F_{1} / F_{0}$ and $V_{1} / V_{0}$ show significant increases at low contrast compared to high contrast (Fig. 5). The distribution of $V_{1} / V_{0}$ ratios presented as a histogram reveal a significant shift toward higher values at low contrasts compared to high contrasts $(n=20, p=0.008$, one-sided $t$ test; Fig. $5 A$, blue symbols). The population spiking responses 

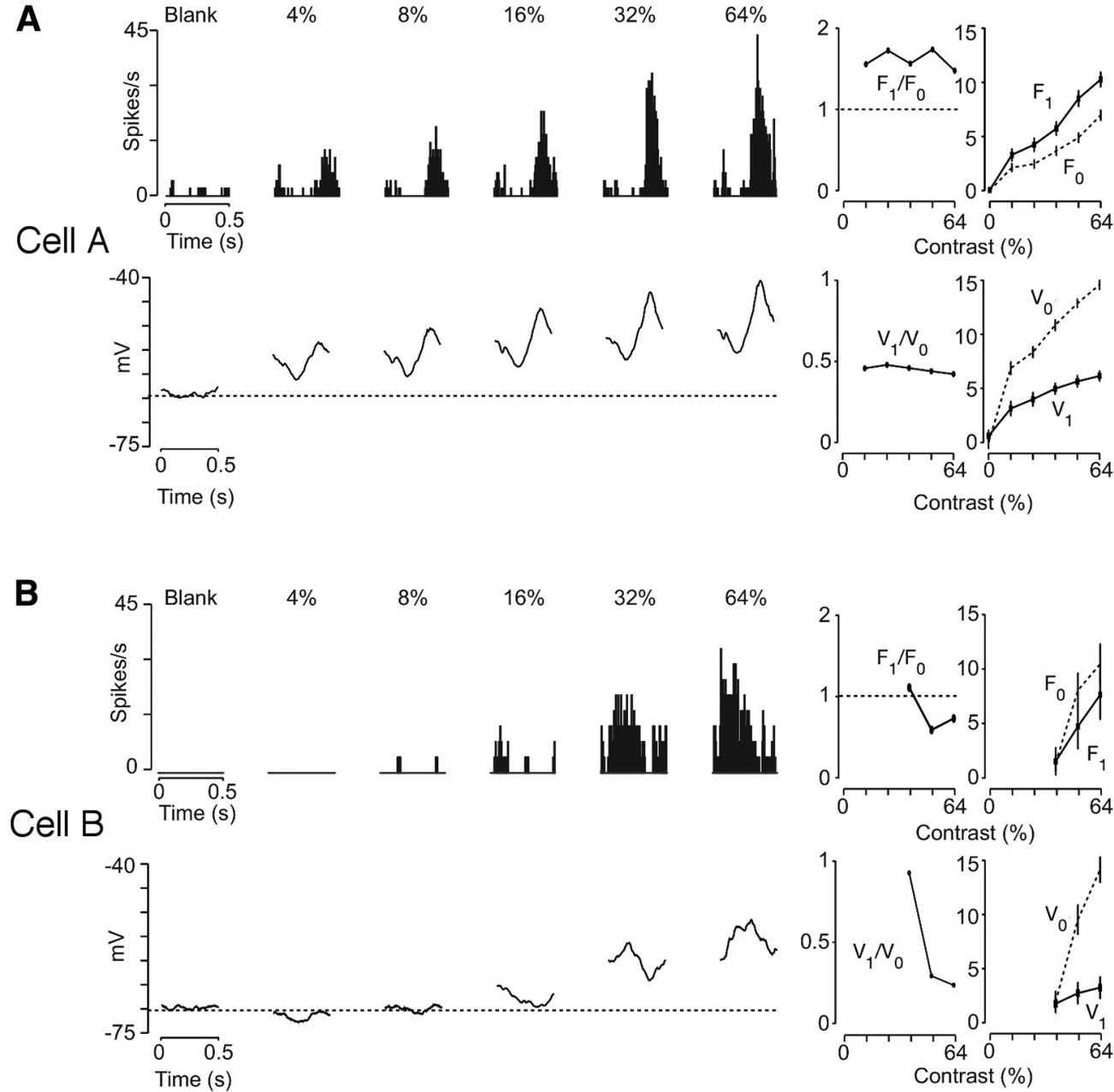

Figure 4. The responses of two example mouse V1 cells (Cell A and Cell B) to drifting gratings $(\boldsymbol{A} \& \boldsymbol{B})$. Left panel: cycle-averaged spiking rate (top panel) and membrane potential (bottom panel) responses to drifting sinusoidal gratings at different stimulus contrasts. From left to right, responses to a blank screen and five stimulus contrasts (blank, 4\%, 8\%, 16\%, 32\% and 64\%) are shown for each cell. The broken lines represent spontaneous spiking rate and resting membrane potential $\left(V_{\text {rest }}\right)$ for spiking rate (top panel) and membrane potential (bottom panel) responses, respectively. Right panel: The spiking modulation ratios $\left(F_{1} / F_{0}\right.$, top panel) and the membrane potential modulation ratios $\left(V_{1} / V_{0}\right.$, bottom panel) plotted as functions of contrast. The unity line, where $F_{1} / F_{0}=1$, is indicated as a broken line in the top panel. $F_{0}$ and $F_{1}$ values (top panel) and $V_{0}$ and $V_{1}$ values (bottom panel) are shown as functions of contrast.

also show significant increases in $F_{1} / F_{0}$ ratios at low contrasts $(n=20, p=0.02$, one-sided $t$ test; Fig. $5 B)$.

\section{Responses to contrast reversing gratings}

An alternative method to quantify the nonlinearities of cortical neurons is to examine the modulated responses to contrast-reversing gratings (Hawken and Parker, 1987). An ideal simple cell should modulate at the TF of the contrast reversal $\left(F_{1}\right)$, and the timing of its response should depend on the spatial phase of the grating (Fig. 6, left). An ideal complex cell should modulate at twice the TF of the contrast reversal $\left(F_{2}\right)$, and the timing of its response should not depend on the spatial phase of the grating (Fig. 6, right). One can then distinguish simple and complex cells by considering the first and second Fourier components in the complex plane (Fig. 6B). Simple cells should have large $F_{1}$ components that lie along an axis in the complex plane. For the example simple cell, that axis is along the abscissa. In contrast, the example complex cell has small $F_{1}$ components, but a large $F_{2}$ component for which the response does not change with the stimulus phase. To extract a metric that describes the relative $F_{1}$ and $F_{2}$ modulations of the responses, we computed the amplitude of the projection of the $F_{1}$ values onto their principle axis in the complex plane, and compared that to the vector average $F_{2}$ value in the complex plane. Doing so enforces the expectation that the timing of the $F_{2}$ component should be invariant to spatial phase. The resulting contrast reversing modulation index $\left(F_{2} / F_{1}\right)$ is large for complex cells and small for simple cells.

To quantify how much $\mathrm{V} 1$ neurons shift to more simplelike behavior as contrast is lowered, we presented contrastreversing gratings at eight different spatial phases and extracted the phase and amplitude of the Fourier components at the TF of the reversing gratings and at twice the TF of the reversing gratings (Fig. 7). These measurements 
A

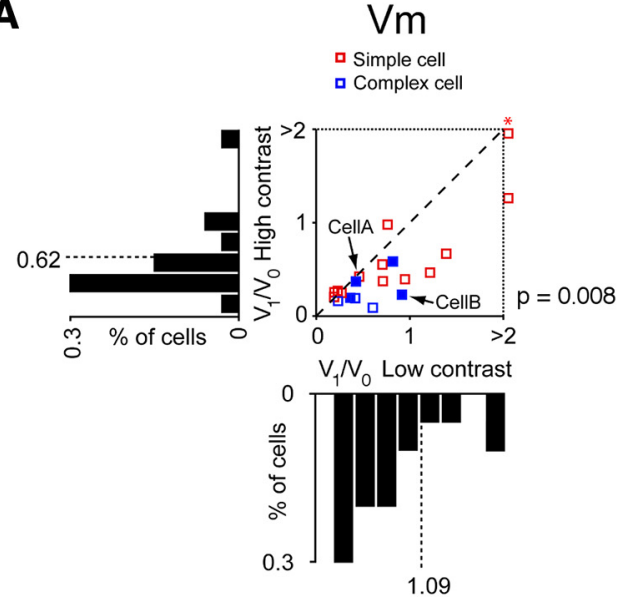

B

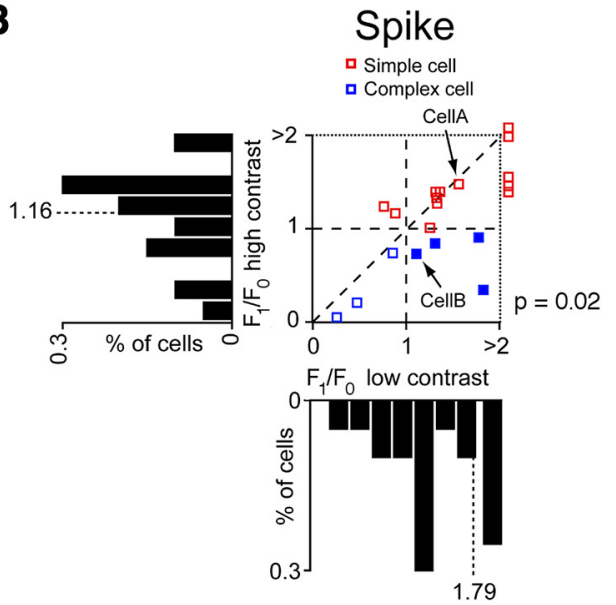

Figure 5. Population results of mouse $V_{1}$ cells recorded with drifting gratings $(n=20)$. A: Scatter plots of membrane potential modulation ratios $\left(V_{1} / V_{0}\right)$ and $B$ : spiking modulation ratios $\left(F_{1} / F_{0}\right)$ at low $(16 \%)$ and high $(64 \%)$ contrasts. The diagonal broken lines indicate the unity lines, where the modulation ratios are the same at high and low contrasts. Compared to high contrasts, the modulation ratios showed significant increases at low contrasts in both membrane potential (one-sided $t$-test, $p=0.008$ ) and spiking rate (one-sided t-test, $p=0.02$ ). Red symbols represent cells that have $F_{1} / F_{0}>1$ (simple cells) at high contrast. Blue symbols represent cells that have $F_{1} / F_{0}<1$ (complex cells) at high contrast. In $B$, the horizontal and vertical broken lines indicate $F_{1} / F_{0}=1$. Data from the example cells in Figure 4 are marked as Cell A and Cell B on both $\boldsymbol{A}$ and $\boldsymbol{B}$. Histograms of distributions of modulation ratios at high and low contrasts are plotted on the same scale next to the corresponding scatter plot axis. The broken lines indicate the means of the distributions. $\left(\boldsymbol{A}: * \mathrm{~V}_{1} / \mathrm{V}_{0}\right.$ at low contrast: $4.55, \mathrm{~V}_{1} / \mathrm{V}_{0}$ at high contrast: 7.22$)$.

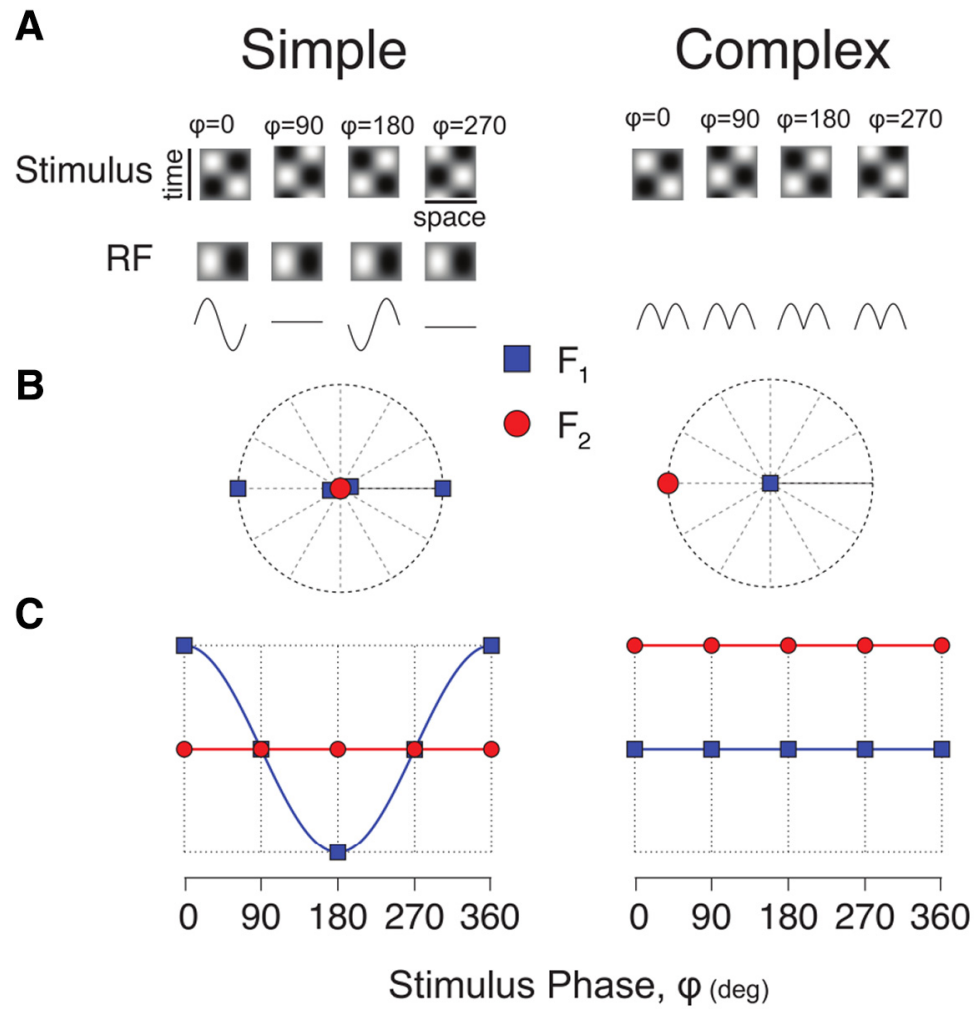

Figure 6. Model responses of a simple and a complex cell to contrast-reversing gratings. $\boldsymbol{A}$, Simple cell models (left) and complex cell models (right) have distinct responses to contrast reversing gratings. Four different spatial phases of the contrast reversing grating are shown in the top row and the receptive fields of the simple cell are shown below. The receptive field for the complex cell is not shown but reflects the quadrature pairs of simple cell receptive fields (Adelson and Bergen, 1985). Model responses to the contrast reversing grating are shown in the third row. $B$, Complex plane representation of the responses of the simple cell (left) and complex cell (right) at the first $\left(F_{1}\right.$, blue square) and second $\left(F_{2}\right.$, red circle) Fourier frequency. $\boldsymbol{C}$, Amplitudes of the $F_{1}$ and $F_{2}$ frequencies projected onto the principle axis (see results). 


\section{Cell C}
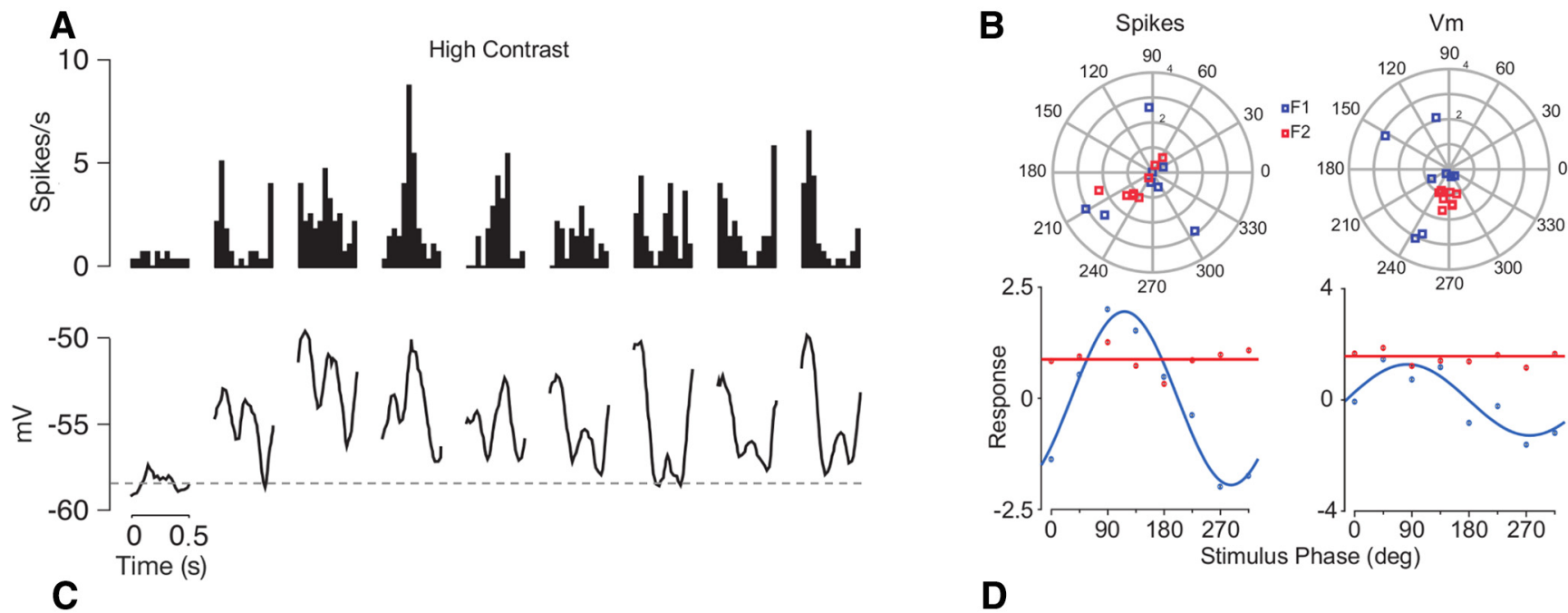

D
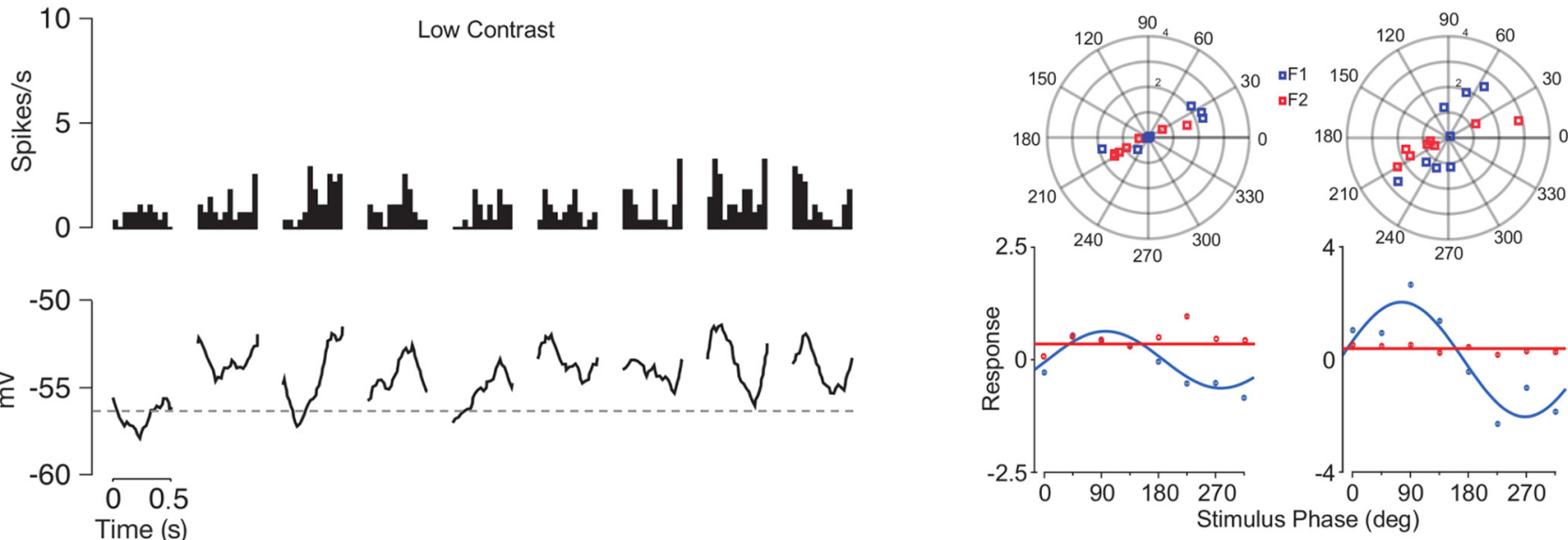

Figure 7. Responses of an example mouse V1 cell (Cell C) to contrast-reversing gratings. $\boldsymbol{A}$, Cycle-averaged spiking rate (top) and membrane potential (bottom) to contrast-reversing gratings at high (100\%) stimulus contrasts for eight different spatial phases. From left to right, responses to a blank screen and spatial phases $0^{\circ}, 45^{\circ}, 90^{\circ}, 135^{\circ}, 180^{\circ}, 225^{\circ}, 270^{\circ}$ and $315^{\circ}$ are shown. $\boldsymbol{B}, \mathrm{F}_{1}(\mathrm{blue})$ and $\mathrm{F}_{2}$ (red) values calculated from spiking rate (left panels) and membrane potential (right panels) are plotted separately as functions of spatial phases for high (100\%) stimulus contrasts. $\boldsymbol{C}$, Similar to $\boldsymbol{A}$, but panels show responses at low (22\%) stimulus contrast. $\boldsymbol{D}$, Similar to $\boldsymbol{B}$ but at low (22\%) stimulus contrast.

were made both for the spiking rate of the neurons and their underlying membrane potentials.

As with drifting gratings we found that reductions in contrast caused systematic changes in membrane potential modulations that reflected a shift toward more simple-like behavior in complex cells. At high contrast these complex cells are characterized by frequency doubled responses in both membrane potential and spiking rate (Fig. $7 A$ ). When contrast was lowered, however, the amplitude of the frequency doubled responses declined relative to the modulation at the TF of the reversing grating. Note that not only do modulations emerge at low contrasts, but the timing of the modulations systematically shifts with the spatial phases of the gratings, as predicted by an ideal simple cell (Fig. 6).

As shown in the membrane potential and spiking rate traces, the projected $F_{2}$ and $F_{1}$ modulations vary across stimulus phase (Fig. 7B). To quantify the changes outlined above for each cell, we estimated individual $F_{1}$ and $F_{2}$ (spikes) and $V_{1}$ and $V_{2}$ (membrane potentials) values across all spatial phases for each stimulus contrast tested. For $F_{2}$ and $V_{2}$, we simply averaged across all stimulus spatial phases since these values were spatial phase invariant (Figs. 6C, 7B, red lines). However, averaging across all stimulus spatial phases does not work for $F_{1}$ and $V_{1}$ because they are spatial phase dependent (Figs. $6 C, 7 B$, blue lines). At high contrasts the amplitudes of the $F_{2}$ and $V_{2}$ modulations do not modulate with spatial phase, while the $F_{1}$ and $V_{1}$ components clearly modulate. When contrast is lowered, the $F_{2}$ and $V_{2}$ modulation amplitudes decline more rapidly relative to the $F_{1}$ and $V_{1}$ components, respectively. These changes cause an overall decline in the membrane potential modulation ratio $\left(V_{2} / V_{1}\right)$ from 0.7 at high contrast to 0.12 at low contrast (Fig. 8A, arrow). As small modulation ratios are associated with simple cells and larger ones with complex cells, this is an example in which contrast shifts the behavior of the neuron toward more simple-like responses. 
A
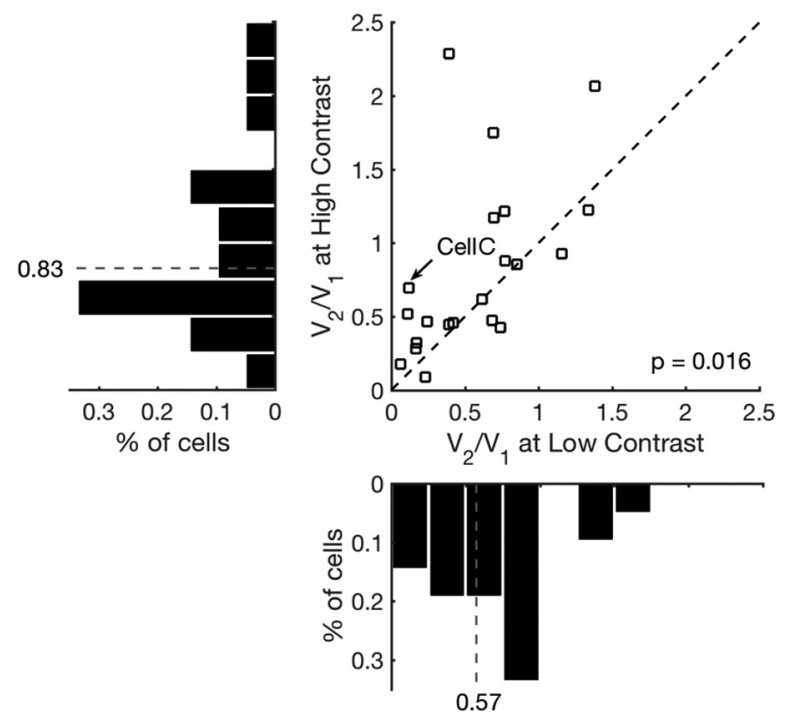

B

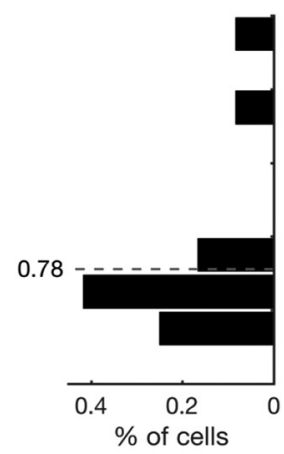

Figure 8. Population results of mouse V1 cells recorded with contrast-reversing gratings. $\boldsymbol{A}, \boldsymbol{B}$, Scatter plots of membrane potential modulation ratios $\left(\mathrm{V}_{2} / \mathrm{V}_{1}\right)(\boldsymbol{A})$ and spiking modulation ratios $\left(\mathrm{F}_{2} / \mathrm{F}_{1}\right)(\boldsymbol{B})$ at low $(22 \%$ or $32 \%)$ and high $(100 \%)$ contrasts. The diagonal broken lines indicate the unity lines, where the modulation ratio is the same at high and low contrasts. In $\boldsymbol{B}$, the horizontal and vertical broken lines indicate $F_{2} / F_{1}=1$. Compared to high contrasts, the modulation ratios showed significant increases at low contrasts in both membrane potential responses $(n=21$, one-sided $t$-test, $p=0.016)$ and spiking rate $(n=12$; one-sided $t$-test, $p=0.021)$. Data from the example cell in Figure 7 is marked as Cell $\mathbf{C}$ on both $\boldsymbol{A}$ and $\boldsymbol{B}$. Histograms of distributions of modulation ratios at high and low contrasts are plotted on the same scale next to the corresponding scatter plot axis. The broken lines indicate the means of the distributions.

To quantify how contrast altered the behavior of our complex cell population we estimated $V_{2} / V_{1}$ and $F_{2} / F_{1}$ modulation indices across our sample population (membrane potential: $n=21$, spikes: $n=12$; Fig. 8). We found that the membrane potential modulation ratio systematically declined with contrast, changing from a mean value of 0.83-0.57 ( $p=0.016$, one-sided $t$ test). There was a similar, but more modest, change in the modulation ratios obtained from spiking rate across our sample population ( $p=0.021$, one-sided $t$ test). Therefore, as with drifting gratings, neurons in mouse visual cortex shift toward more simple-like responses as contrast is lowered.

\section{Discussion}

Contrast-dependent phase sensitivity has been documented in a subpopulation of neurons in V1 of cat (Crowder et al., 2007; van Kleef et al., 2010; Hietanen et al., 2013) and primate (Henry and Hawken, 2013; Cloherty and Ibbotson, 2015). The current study demonstrates that contrast-dependent phase sensitivity is also present in the V1 of mouse. Cortical visual processing in mice has been studied extensively in the past decade (Niell and Stryker, 2008; Huberman and Niell, 2011; Tan et al., 2011). The abundant opportunities for genetic manipulation have made mouse visual cortex a useful model in addition to carnivores and primates for studying RF properties (Wang et al., 2006; Liu et al., 2009; Zariwala et al., 2011). All previous literature showing contrastdependent changes in response linearity in cats and monkeys has been quantified using modulation ratios calculated from extracellular responses to drifting sinusoidal gratings
(Crowder et al., 2007; van Kleef et al., 2010; Henry and Hawken, 2013; Hietanen et al., 2013; Cloherty and Ibbotson, 2015) or contrast-reversing gratings (Meffin et al., 2015).

We used whole-cell recordings to shed light on how complex cells emerge in V1. A simple model to describe this process is that recurrent cortical connectivity between neurons with distinct spatial selectivity generates the spatial-invariant responses that characterize complex cells. Two models have been proposed to describe this shift, one from Hubel and Wiesel in which simple cells receive inputs from the dorsal lateral geniculate nucleus (dLGN) in the thalamus and converge onto complex cells in one step to generate spatial invariance (Hubel and Wiesel, 1962). Alternatively the generation of spatial invariance may require many steps, which reflect an increase in the proportion of cortical circuitry that neurons receive (Chance et al., 1999). One way to distinguish these possibilities is to observe how input strength alters the emergence of complex cells. Mouse dLGN neurons show mostly linear contrast sensitivity, the responses of individual dLGN cells increase with increasing contrasts (Grubb and Thompson, 2003; Tang et al., 2016). Lien and Scanziani (2013) have shown that recurrent cortical excitation to simple cells in mouse V1 is phase-sensitive and matches the dLGN inputs. However, it is unclear whether this is the case for complex cells. We demonstrate that as contrast declines both membrane potential and spiking modulation ratios increase, as expected from the recurrent model proposed by Chance et al. (1999). 
An alternative explanation for the shift in modulation ratio at low contrast is the variability of contrast response curves across simple cells. Neurons within V1 vary in the contrast at which they saturate, such that for some neurons the changes in contrast may yield large changes in response amplitude whereas for others they may evoke little effect (Van den Bergh et al., 2010). A simple model that includes the variance in the contrast response curves of simple cells which converge onto a complex could only account for input modulation ratio shifts of $<0.1$, relative to our measures of modulation ratio shifts of $>0.45$ (data not shown).

We analyzed the responses to drifting gratings and found that there is a shift in the input modulation ratio $\left(V_{1} N_{0}\right)$ with contrast consistent with a synaptic model (mean $V_{1} / V_{0}$ high contrast: 0.62 , low contrast: 1.09). While the threshold nonlinearity may play a role in altering the phase sensitivity of neurons (Priebe et al., 2004), there is a clear synaptic component to the shift in phase sensitivity. Also, as expected, when stimulated with high-contrast reversing gratings these cells exhibited various degrees of frequency-doubled responses (Meffin et al., 2015). However, at low contrasts, the same cells showed more modulated, phase sensitive responses to drifting gratings and a tendency to respond to selected spatial phases during stimulation with contrast-reversing gratings.

In summary, for some years now it has been noted that complex cells show increased modulatory responses at low contrasts, suggesting that they are more phase sensitive at low contrasts (Crowder et al., 2007; van Kleef et al., 2010; Henry and Hawken, 2013; Cloherty and Ibbotson, 2015; Meffin et al., 2015). We demonstrate that this is not simply a manifestation of the iceberg phenomenon (Carandini and Ferster, 2000; Mechler and Ringach, 2002; Priebe et al., 2004), but instead a systematic shift in the inputs that cortical neurons receive. This network level change in input modulation with contrast is consistent with a model for the generation of invariant responses in which complex cells emerge steadily through the cortical network through increases in the degree of recurrent inputs that they receive.

\section{References}

Adelson EH, Bergen JR (1985) Spatiotemporal energy models for the perception of motion. J Opt Soc Am A 2:284-299.

Ben-Yishai R, Bar-Or RL, Sompolinsky H (1995) Theory of orientation tuning in visual cortex. Natl Acad Sci USA 92:3844-3848.

Carandini M, Ferster D (1997) A tonic hyperpolarization underlying contrast adaptation in cat visual cortex. Science 276:949-952.

Carandini M, Ferster D (2000) Membrane potential and firing rate in cat primary visual cortex. J Neurosci 20:470-484.

Chance FS, Nelson SB, Abbott LF (1999) Complex cells as cortically amplified simple cells. Nat Neurosci 2:277-282.

Cloherty SL, Ibbotson MR (2015) Contrast-dependent phase sensitivity in V1 but not V2 of macaque visual cortex. J Neurophysiol 113:434-444.

Crowder NA, van Kleef J, Dreher B, Ibbotson MR (2007) Complex cells increase their phase sensitivity at low contrasts and following adaptation. J Neurophysiol 98:1155-1166.
De Valois RL, Albrecht DG, Thorell LG (1982) Spatial frequency selectivity of cells in macaque visual cortex. Vision Res 22:545559.

Douglas RJ, Koch C, Mahowald M, Martin KAC, Suarez HH (1995) Recurrent excitation in neocortical circuits. Science 269:981-985.

Ferster D, Jagadeesh B (1992) EPSP-IPSP interactions in cat visual cortex studied with in vivo whole-cell patch recording. J Neurosci 12:1262-1274.

Gilbert CD (1977) Laminar differences in receptive-field properties of cells in cat primary visual-cortex. J Physiol 268:391-421.

Grubb MS, Thompson ID (2003) Quantitative characterization of visual response properties in the mouse dorsal lateral geniculate nucleus. J Neurophysiol 90:3594-3607.

Hammond P, Ahmed B (1985) Length summation of complex cells in cat striate cortex - a reappraisal of the special standard classification. Neuroscience 15:639-649.

Hawken MJ, Parker AJ (1987) Spatial properties of neurons in the monkey striate cortex. Proc R Soc Lond B Biol Sci 231:251288.

Henry CA, Hawken MJ (2013) Stability of simple/complex classification with contrast and extraclassical receptive field modulation in macaque V1. J Neurophysiol 109:1793-1803.

Henry GH (1977) Receptive-field classes of cells in striate cortex of cat. Brain Res 133:1-28.

Hietanen MA, Cloherty SL, van Kleef JP, Wang C, Dreher B, Ibbotson MR (2013) Phase sensitivity of complex cells in primary visual cortex. Neuroscience 237:19-28.

Hubel DH, Wiesel TN (1962) Receptive fields, binocular interaction and functional architecture in the cat's visual cortex. J Physiol 160:106-154.

Huberman AD, Niell CM (2011) What can mice tell us about how vision works? Trends Neurosci 34:464-473.

Jagadeesh B, Wheat HS, Kontsevich LL, Tyler CW, Ferster D (1997) Direction selectivity of synaptic potentials in simple cells of the cat visual cortex. J Neurophysiol 78:2772-2789.

Lien AD, Scanziani M (2013) Tuned thalamic excitation is amplified by visual cortical circuits. Nat Neurosci 16:1315-23.

Liu BH, Li P, Li YT, Sun YJ, Yanagawa Y, Obata K, Zhang LI, Tao HW (2009) Visual receptive field structure of cortical inhibitory neurons revealed by two-photon imaging guided recording. J Neurosci 29:10520-10532.

Maffei L, Fiorenti A (1973) The visual cortex as a spatial frequency analyzer. Vision Res 13:1255-1267.

Margrie TW, Brecht M, Sakmann B (2002) In vivo, low-resistance, whole-cell recordings from neurons in the anaesthetized and awake mammalian brain. Pflugers Arch 444:491-498.

Martinez LM, Wang Q, Reid RC, Pillai C, Alonso JM, Sommer FT, Hirsch JA (2005) Receptive field structure varies with layer in the primary visual cortex. Nat Neurosci 8:372-379.

Mechler F, Ringach DL (2002) On the classification of simple and complex cells. Vision Res 42:1017-1033.

Meffin H, Hietanen MA, Cloherty SL, Ibbotson MR (2015) Spatial phase sensitivity of complex cells in primary visual cortex depends on stimulus contrast. J Neurophysiol 114:3326-3338.

Movshon JA, Thompson ID, Tolhurst DJ (1978) Spatial and temporal contrast sensitivity of neurones in areas 17 and 18 of cat's visual cortex. J Physiol 283:101-120.

Niell CM, Stryker MP (2008) Highly selective receptive fields in mouse visual cortex. J Neurosci 28:7520-7536.

Priebe NJ, Mechler F, Carandini M, Ferster D (2004) The contribution of threshold to the dichotomy of cortical simple and complex cells. Nat Neurosci 7:1113-1122.

Ringach DL, Shapley RM, Hawken MJ (2002) Orientation selectivity in macaque V1: diversity and Laminar dependence. J Neurosci 22:5639-5651.

Skottun BC, De Valois RL, Grosof DH, Movshon JA, Albrecht DG, Bonds AB (1991) Classifying simple and complex cells on the basis of response modulation. Vision Res 31:1079-1086. 
Somers DC, Nelson SB, Sur M (1995) An emergent model of orientation selectivity in cat visual cortical simple cells. J Neurosci 15: 5448-5465.

Spitzer H, Hochstein S (1988) Complex-cell receptive-field models. Prog Neurobiol 31:285-309.

Tan AY, Brown BD, Scholl B, Mohanty D, Priebe NJ (2011) Orientation selectivity of synaptic input to neurons in mouse and cat primary visual cortex. J Neurosci 31:12339-12350.

Tang JY, Jimenez SCA, Chakraborty S, Schultz SR (2016) Visual receptive field properties of neurons in the mouse lateral geniculate nucleus. PLoS One 11:e0146017.

Van den Bergh G, Zhang B, Arckens L, Chino YM (2010) Receptive field properties of $\mathrm{V} 1$ and $\mathrm{V} 2$ neurons in mice and macaque monkeys. J Comp Neurol 518:2051-2070. van Kleef JP, Cloherty SL, Ibbotson MR (2010) Complex cell receptive fields: evidence for a hierarchical mechanism. J Physiol 588: 3457-3470.

Wang KH, Majewska A, Schummers J, Farley B, Hu C, Sur M, Tonegawa $S$ (2006) In vivo two-photon imaging reveals a role of arc in enhancing orientation specificity in visual cortex. Cell 126: 389-402.

Williams PE, Shapley RM (2007) A dynamic nonlinearity and spatial phase specificity in macaque V1 neurons. J Neurosci 27:57065718.

Zariwala HA, Madisen L, Ahrens KF, Bernard A, Lein ES, Jones AR, Zeng $H$ (2011) Visual tuning properties of genetically identified layer 2/3 neuronal types in the primary visual cortex of cretransgenic mice. Front Syst Neurosci 4:162 